\title{
Sinus-Milieus - ein Instrument für die Redaktionsarbeit im Fernsehen?
}

Morgens um sieben ist die (Fernseh-)Welt noch in Ordnung, doch spätestens um neun Uhr gerät sie in Bewegung oder gar aus den Fugen. Denn dann werden die Quoten des vergangenen Tages veröffentlicht, und ein Blick auf die Zahlen entscheidet über die Stimmung in Redaktionszimmern und Chefetage. Haben die Sendungen die Zuschauer erreicht? Sind die Erwartungen an bestimmte Programme erfüllt worden? Konnte sich das Experiment durchsetzen auf dem Markt? Und wie haben Gegenprogramm und Programmumfeld die Marktanteile beeinflusst? Sind wir Tagessieger geworden oder weit abgeschlagen? Und wo waren die jungen Zuschauer? Alltägliche Fragen, die neben der inhaltlichen Programmkritik einen breiten Raum einnehmen und über das Wohl und Wehe von Sendereihen und Redaktionen entscheiden können.

Die Fixierung auf die Quoten wird uns Fernsehmachern oft vorgeworfen, vor allem den Kolleginnen und Kollegen in den öffentlich-rechtlichen Sendern. „Das habt Ihr doch nicht nötig, Ihr braucht doch keine Werbeeinnahmen wie die Privaten", heißt es dann. Aber wer zahlt schon gerne Gebühren für ein Programm, das ihm nicht gefällt und das er nicht nutzt? Eine schlechte Quote und abfallende Kurven bedeuten ja nichts anderes als dass König Zuschauer kein Interesse an dieser Sendung hatte oder mehr Interesse für parallel ausgestrahltes Programm. Wer über Quoten redet, spricht daher immer von unserem Souverän und seinem Einschaltverhalten, das sich in den Zahlen der Quoten und der Statistiker niederschlägt. Wie die Absatzzahlen eines Buches oder die Klicks auf eine Internetseite Indikatoren für die geweckte Aufmerksamkeit und ein gutes Marketing sind, aber noch kein Garant für Qualität, so besagen die Quoten einer Fernsehsendung etwas über das generelle Zuschauerinteresse, mehr aber auch nicht. Andere Methoden müssen die Analysen ergänzen.

In der Medienforschung des ZDF sind die Sinus-Milieus bereits gute alte Bekannte. Seit vielen Jahren gehen die Erhebungen über die klassischen Fragen nach Alter, Geschlecht und Bildungsgrad hinaus, um die Milieus und Lebensstile einzubeziehen. Die Frage, wofür sich unsere Zuschauer interessieren und welches Potential einzelne Sendeformen haben, ist von zentraler Bedeutung für die Planung von Programmen und Programmschemata. So wird in regelmäßigen Abständen genaues Material erhoben zur Akzeptanz von Sendungen, das über die Auswertung der allfälligen Quoten der Gesellschaft für Konsumforschung hinausgeht. 
Und dies nicht nur für die wichtigen Zugpferde des Programms in der Prime-Time, auch Sendungen an den Programmrändern und im Tagesprogramm werden auf den Prüfstein der Medienforschung gelegt.

Für die beiden wichtigsten Sendereihen der Redaktionen Kirche und Leben im ZDF, „sonntags“ und „37 “ hat das Heidelberger Institut „psyma" 2004 qualitative Studien gemacht. Für die Gottesdienstübertragungen als Serviceangebot ist dank immer noch steigender Zuschauerzahlen eine solch ausführliche Studie bisher nicht erfolgt. Dennoch liegen auch für sie Angaben zu den Sinus-Milieus vor.

Wie passen die Konzepte der Sendungen mit der Rezeption in den einzelnen Milieus zusammen? Sind die Überlegungen der Redaktion kompatibel mit den Bedürfnissen der Zuschauer?

\section{Die Sendung "sonntags"}

Die Sendung "sonntags - TV fürs Leben“ wird jeden Sonntag von 9.02 Uhr (nach den heute-Nachrichten) bis $9.30 \mathrm{Uhr}$ ausgestrahlt. Sie wird von den beiden Redaktionen Kirche und Leben evangelisch und katholisch gemeinsam verantwortet, nicht im Wechsel, sondern von einem Redakteurspool, der bewusst ökumenisch zusammengesetzt ist. Es handelt sich um ein moderiertes Magazin, dessen Themen sich um Ethik, Religion, Kirche, um Fragen der Lebensgestaltung, aber auch um Boulevard, Kultur und Gesellschaft drehen. Die Sendung „sonntags“ versteht sich als Werte-Magazin mit einem besonderen Blick auf die jeweiligen Themen. Wichtig ist den Redaktionen bei der Wahl der Beiträge der spezielle Zugang, der andere Blick und die positive Grundstimmung, die z.B. durch eine feststehende Rubrik „Menschen und Projekte“ erzeugt wird. Hier werden Menschen vorgestellt, die in besonderer Weise die Initiative ergriffen haben, etwas für die Gesellschaft tun und sich ehrenamtlich engagieren. Weitere Rubriken sind „Das aktuelle Stück“, „Der Ausflug“, der "Tipp der Woche“ von Werner Küstenmacher und das Schwerpunktthema, das in Beiträgen und im Gespräch mit einem Studiogast aus unterschiedlichen Perspektiven aufgearbeitet wird.

Überrascht waren die Forscher von der besonderen Zielgruppe der Sendung. Bei der Auswahl war ein Interesse an ethischen und religiösen Fragestellungen vorausgesetzt worden. Diese Gruppe unterscheidet sich vom Gros der Fernsehzuschauer durch ihr Engagement, ihr ausgewähltes Rezeptionsverhalten und ihren sensiblen, aber auch selbstbewussten und intensiven Umgang mit ihren Mitmenschen. Eine Nebenher-Rezeption spielt für sie eine untergeordnete Rolle, sie suchen sich Sendungen aus, durch die sie gefordert werden, sie wollen sich auf das Geschehen am Bildschirm einlassen. 
Von den Sinus-Milieus entspricht diese Gruppe vor allem den Konservativen und Traditionsverwurzelten, also eher dem Segment A; aber auch die Postmateriellen werden erreicht. $\mathrm{Zu}$ den Merkmalen der Konservativen gehören das „Bewahren der Werte, Traditionen und bewährten In stitutionen" und die Pflege des familiären, kulturellen und nationalen Erbes sowie der sozialen Verantwortung. Die Philosophie von „sonntags“, positive Beispiele von Engagement zu zeigen und die werthaltigen Aspekte eines Themas aufzuarbeiten, entspricht also sehr genau den Vorstellungen dieser Zielgruppe. Die Konservativen wünschen sich, laut Studie, eine praktische Umsetzung der Ökumene und das Vorantreiben des interchristlichen Dialogs - auch diesen Anliegen kommt die ökumenische Ausrichtung des Magazins entgegen.

\section{Die Sendung „37 $37^{\circ \Perp}$}

Eine andere Zielgruppe spricht die Sendung „37 “ an. Sie wird jeweils dienstags um 22.15 Uhr im ZDF ausgestrahlt und verantwortet von drei Redaktionen: Kirche und Leben evangelisch, Kirche und Leben katholisch, Geschichte und Gesellschaft. Ein regelmäßiger Runder Tisch aller Beteiligten sorgt dafür, dass ein gemeinsames Format entwickelt und eingehalten wird. Die Sendetermine sind entsprechend verteilt und einzelnen Redaktionen zugeordnet, die ihre Sendungen unabhängig verantworten. Das Format erzählt Geschichten von Menschen, deren Lebenswegen, Schicksalen und gesellschaftlicher Situation. Der Titel „37 “ leitet sich von menschlicher Körpertemperatur an der Grenze zur Fieberschwelle ab und ist Programm: Es geht um existentielle Fragen des Alltags oder um Ausnahmesituationen, die aber dennoch einen Bezug zum Zuschauer haben. Die Sendung ist bewusst subjektiv, die jeweiligen Protagonisten gewähren einen Einblick in ihr Leben und werden respektvoll und behutsam behandelt. Eine wertende Haltung soll bewusst vermieden, eher Empathie geweckt werden.

Für die Rezeption bedeutet dies, dass die Seher „37 “ eine große Nähe und Emotionalität zusprechen, das Format bei hoher Identifikation aber auch Distanz ermöglicht, eine Kombination, die den Befragten sehr wichtig war.

Die Sendung erreicht auch ein jüngeres Publikum, die Sinus-Milieus sind eher anders verteilt. Für „37 “ interessieren sich vor allem die Postmateriellen (17 Prozent) und die Konservativen (16,7 Prozent), aber auch die Traditionsverwurzelten (12,4 Prozent); die Etablierten (11,6 Prozent) und die Bürgerliche Mitte (10,0 Prozent) schätzen nahezu in gleicher Weise die Sendungen. Gründe für die Vorliebe der Postmateriel- 
len für „37 “ liegen z.B. in deren Sehnsucht nach Authentischem, ihrem Ideal eines aufgeklärten Individuums mit ganzheitlichem Lebensentwurf; dieser Disposition wird die betont subjektive Darstellung von Menschengeschichten gerecht.

\section{Gottesdienstübertragungen}

Die sonntäglichen Gottesdienste im ZDF, jeweils von 9.30 bis $10.15 \mathrm{Uhr}$ und im Wechsel evangelisch oder katholisch, sprechen im Gegensatz zu „37 “ ein kirchlich orientiertes Publikum an. Mit knapp einer Million Zuschauer im Durchschnitt hat die Sendung eine klare Zielgruppe, die relativ treu ist und eigens für die Übertragungen einschaltet. Viele alte und kranke Menschen nutzen den Fernsehgottesdienst, weil sie das Haus nicht verlassen können, um selbst in die Kirche zu gehen. Daneben gibt es Menschen, die das niederschwellige Angebot nutzen, um am Gottesdienst teilzunehmen. Im TV können sie, ohne in der Gemeinde vor Ort aufzufallen, hereinschnuppern, aber auch wieder ausschalten, wenn es ihnen nicht gefällt.

Erwartungsgemäß zeigt die Auswertung nach Sinus-Milieus ein deutliches Übergewicht bei den Konservativen (40,8 Prozent) und den Traditionsverwurzelten (31,8 Prozent). Erstaunlicher ist dagegen, dass gemessen am Gesamtdurchschnitt der Gottesdienste, der 2005 einen Marktanteil von 13,3 Prozent erreicht hat, auch die Hedonisten mit 14,3 Prozent überdurchschnittlich vertreten sind. Ein Milieu, dem man eher Interesse an kurzfristigem Lustgewinn zutraut, das für die modernen Missionsstrategen eher unattraktiv erscheint. Die Sinus-Milieustudie zeigt aber auch, dass diese Gruppierung ebenfalls die modernen Sinnsucher erfasst, und diese scheinen sich zu einem gewissen Prozentsatz von einem Angebot wie Fernsehgottesdiensten Anregungen und Impulse zu versprechen. Die Niederschwelligkeit des Fernsehens ermöglicht Kontakte zu einer Form des spirituellen Angebotes, das in der realen Situation einer Pfarrgemeinde kaum genutzt werden würde.

\section{Was kann man lernen?}

Bei einer Diskussion der Sinus-Milieustudie und ihrer Konsequenzen für die Arbeit der Katholischen Verbände und Organisationen wurde sehr deutlich festgestellt, dass es keine Rezepte gibt, wie man z.B. die Gruppe der Experimentalisten für die Verbandsarbeit gewinnen kann, vor allem, wenn man die Traditionsverwurzelten nicht verlieren will. Bestimmte 
Milieus schließen sich aus in der Art und Weise, wie sie angesprochen werden wollen. Und wer strategisch denkt, der sollte sich vor allem darum kümmern, wie sich über die Jahre die Größenordnüngen der einzelnen Milieus verändert haben. Es macht wenig Sinn, Fantasie zu entwickeln, wie man das Milieu der DDR-Nostalgischen erschließen kann. Seit den 90er Jahren ist dieses Milieu auf knappe 6 Prozent geschrumpft, und man muss kein Soziologe sein um zu prognostizieren, dass es auch in den nächsten Jahren weiter schwinden wird. Anstrengungen lohnen sich dagegen im Milieu der Postmateriellen, die einerseits eine gewisse Aufgeschlossenheit religiösen Fragen gegenüber zeigen und andererseits kontinuierlich zugenommen haben. Einen besonderen Blick gilt es auf die jungen Menschen zu richten. Deren Ästhetik und Lebensvorstellungen müssen sich widerspiegeln in den Angeboten kirchlicher (Medien-)Arbeit, wenn man sie gewinnen will.

Ein geschlossenes katholisches Milieu gibt es schon lange nicht mehr, und die Sinus-Studie beweist, dass die unterschiedlichen Lebensstile der Menschen unterschiedliche Antworten und Angebote erfordern. Auch ein Massenmedium wie das Fernsehen kann mit einer bestimmten Sendung nie alle Milieus erreichen. Durch eine klare Formatierung und den Zuschnitt auf ein mögliches Zielpublikum versuchen die Macher, ein möglichst großes Marktsegment zu erzielen. Will man die modernen Performer als Publikum, kann dies wohl kaum mit Volksmusiksendungen und Heimatfilmen gelingen. Aber dass Programmgenres dieser Art nicht jedermanns Sache sind und vor allem junge Leute nicht zum Einschalten bringen, weiß der Programmmacher auch ohne aufwändige Untersuchungen von Sinus-Milieus. Die konkrete Redaktionsarbeit, die Auswahl von Themen für „37 “ oder „sonntags“, lebt auch weiterhin vom Bauchgefühl und Instinkt erfahrener Redakteure. Sie brauchen den richtigen Riecher für Fragen, die die Zuschauer interessieren. Es hilft ihnen aber zu wissen, welche Themen nur schwer zu vermitteln sind, wofür sich nur kleine Gruppen begeistern lassen. Und es kann nützlich sein, um Strategien zu entwickeln und neue Potenziale anzustreben.

In den letzten Jahren sind die Marktanteile aller drei Sendeformen der Kirchenredaktionen im ZDF kontinuierlich gestiegen. Der Erfolg ist unter anderem darauf zurückzuführen, dass die Engführung klassischer Kirchenfunkprogramme aufgegeben wurde und die Sendungen auch attraktiv sind für Nichtkirchgänger. Der Preis, den es zu zahlen gilt, ist der Verlust eines bestimmten eng konfessionell-institutionellen Themensektors. Im Sinne der Zukunftsfähigkeit des Programms glauben wir aber, auf dem richtigen Weg zu sein, und die Milieustudie und die Zuschauer geben uns recht. 